\title{
Spatial Reuse in Wireless Ad-hoc Networks
}

\author{
Xingang Guo*, Sumit Roy**, W. Steven Conner* \\ *Network Architecture Lab \\ **Communication and Interconnect Technology Lab \\ Intel Corporation \\ $2111 \mathrm{NE} 25^{\text {th }}$ Ave., JF3-206 \\ Hillsboro, OR 97124 \\ \{xingang.guo, sumit.roy, w.steven.conner\}@intel.com
}

\begin{abstract}
When investigating co-channel spatial reuse, typical studies on ad-hoc networks have adopted static, device-specific transmission/interference ranges regardless of the runtime environment. In this paper, we present an analytical framework to investigate co-channel spatial reuse in dense wireless ad-hoc networks based on RF propagation models for some common network topologies. We derive the minimum separation between simultaneous co-channel transmitters while maintaining desirable signal-noise-interference-ratio at receivers. Spatial reuse is characterized by the ratio between co-channel transmitter (T-T) and transmit-receiver (T-R) distances. Our results show that increasing transmission power improves spatial reuse in ambient noise dominated environments. However, in co-channel interference limited scenarios, increasing transmission power has little effect on spatial reuse. On the other hand, more simultaneous transmissions can be physically possible by decreasing the average $T-R$ separation, independent of transmission power. Our results confirm that the popular practice of using static transmission/interference ranges leads to an oversimplified model that is unable to accurately characterize the spatial reuse (and consequently aggregate network capacity) in such ad-hoc networks.
\end{abstract}

\section{Introduction}

Increasingly wireless ad-hoc networks are used to provide connectivity to devices in environments where engineered network infrastructures don't exist or are expensive to deploy. These networks typically include both 802.11 based ad-hoc networks [2][11][13] and low-power sensor networks [1][19]. Some sensor networks [5][19] must deal with hundreds to thousands of network devices in a confined indoor environment. The proliferation of wireless-enabled mobile computing and consumer electronic devices will also increase node density of 802.11 WLAN-type ad-hoc networks. It has been observed that such dense networks are often saturated with data traffic [24]. Therefore, the data transport capacity is a key performance metric in dense adhoc networks.

The aggregate data transport capacity of such a network is proportional to the number of simultaneous communications it can support. While non-interfering channels are typically created by partitions of time, frequency, code, etc., cochannel reuse in spatially separated locations is a key to determining aggregate capacity, especially in co-channel interference limited environments [21].

Frequency reuse and capacity planning have been extensively studied for cellular networks [12][15][18] where the locations of base stations are known a priori. Hence coordinated channel partitioning among cells (i.e. effectively an FDMA overlay) yields a predictable and managed interference environment. However, the absence of engineered infrastructure in ad-hoc networks coupled with node mobility (leading to nodes joining and leaving the network intermittently) makes it very difficult to implement any such centralized or semi-centralized channel allocation schemes designed for cellular networks, and make them efficient and scalable [6][22].

Without pre-planning, nodes in a wireless ad-hoc network rely on detect-and-transmit schemes to discover the (re)usable channels. A representative method is the CSMA/CA algorithm that forms the basis of the Distributed Coordination Function (DCF) multiple access protocol in 802.11 [11]. A node defers attempting channel access for a (random) period upon detecting either a busy channel or potential collision from one of its transmissions. There is considerable interest in determining how the performance of such MAC algorithms scales spatially in a multi-hop, multicell network.

Runtime link-layer interference has been studied extensively for wide-area macro cellular networks [14][20]. These studies typically only consider interference from the first ring of co-channel cells in a regular frequency reuse pattern, neglecting contributions from secondary cells. In a dense adhoc network, the above assumption is no longer valid. Therefore, a new interference model is needed to calculate the runtime interference and estimate the amount of spatial reuse feasible for indoor, dense ad-hoc networks. 
This paper provides results for regular 1-D and 2-D network topologies using a link-layer based model for the runtime signal-noise-interference ratio (SNIR) at an intended receiver. For a given minimal SNIR, we determine the minimum spatial separation between neighboring transmitters (T-T) as function of T-R distance to characterize the amount of spatial reuse. In section II, we describe our assumptions regarding communication model and network architecture. In section III and IV we present the spatial reuse models for 1-D and 2-D network topologies, respectively. Section V presents figures and discussions on the spatial reuse models. Then we discuss related works in section VI. Section VII concludes the paper.

\section{Wireless Network Model}

In a wireless network, a transmitter can potentially contribute interference to all receivers, consequently affecting their reception. Hence we need realistic models that not only quantify physical layer interference but also capture the interactions of higher layers of the network stack that determine when and where such interference occurs.

\section{A. Physical layer assumptions}

Current studies on ad-hoc networks have largely used the range model [2][16] to characterize wireless links. This simplistically assumes a fixed (radio) transmission range within which perfect communication is possible, and outside which no communication is possible. Such a model does not consider multiple accesses. It ignores the physical reality of a wireless link where a receiver sees contributions from multiple simultaneous interfering sources and is thus inadequate for our purpose.

We adopt instead a link-budget-inspired SNIR model $[4][8][10][12][17][23]$ wherein a communication is successful (with high probability) if and only if the average SNIR at the receiver is above a threshold. This model emphasizes that it is not the absolute received signal strength, but the relative signal strength with respect to the interference (and noise) that determines whether a reception succeeds or not.

A typical path loss model expresses the received strength (in $\mathrm{dB})$ as a function of the T-R separation distance $[10][21][23]$. The signal strength is expressed as a lognormally distributed random variable, i.e.

$$
\begin{aligned}
& P_{R x}(d)=\overline{P_{0}}+10 \gamma \log _{10}\left(\frac{d}{d_{0}}\right)+20 \log _{10}\left(\frac{c}{4 \pi f}\right)+X_{\sigma} \\
& =A+10 \gamma \log _{10}\left(\frac{d}{d_{0}}\right)
\end{aligned}
$$

where $d$ is the T-R separation distance. $d_{0}$ is the reference distance (usually 1 meter). $P_{0}$ is the decibel receiving power at the reference distance. $c$ is the speed of light. $f$ is the center frequency of the RF signal. $\gamma$ is the pathloss exponent indicating how quickly the RF signal decays as distance increases, and has a negative value. Finally $X_{\sigma}$ is a zero-mean Gaussian distributed random variable with standard deviation $\sigma$.

Normally the parameters are calculated from regression analysis of field measurements. The type of the radio, as well as the propagation environment, translates into different values for the pathloss exponent. Table 1 lists typical measurements for some indoor wireless networks [3],

\begin{tabular}{|l|l|l|}
\hline & $802.11 \mathrm{~b}$ & $802.11 \mathrm{a}$ \\
\hline LOS & -1.91 & -1.83 \\
\hline NLOS (typical) & -3.73 & -4.70 \\
\hline
\end{tabular}

Table 1. Pathloss exponents in indoor environments

\section{B. Network architecture assumptions}

To study the essence of spatial reuse, we assume the following,

1. Homogeneous, backlogged nodes: every node has identical receiving sensitivity, transmission power and SNIR threshold. Furthermore, a node always has packets to transmit.

2. Work-conserving data links: no data link will ever be idle. Here we essentially assume a zero-overhead link management (MAC) layer with global knowledge of link availability. Combined with the previous property, the network is always saturated with traffic. Therefore it also generates the worst-case interference.

3. Uniform transmission and propagation environment: RF signal emitted by every node presents the same pathloss characteristic.

4. Uniform node density: hence the average inter-node distance is a constant throughout the network.

5. A node only communicates with its immediate neighbors using unicast. Because of the uniform inter-node distance, the received strength of the respective signal is the same at all receivers.

We also define a symmetric network as one in which every node perceives the same network topology and itself being the center of the network. A significant property of a symmetric, omni-centric network is that there is no edge effect.

\section{Chain Network}

In a symmetric chain network, an infinite number of nodes are placed at equal inter-node distance along a straight line. Let us assign each node a unique id that resembles the integer axis ranging from $-\infty$ to $+\infty$. Without loss of generality, let us consider node 0 receiving, see Figure 1 . In order for 
the communication to succeed, the average SNIR at node 0 must be higher than a threshold, let $S_{0}$ denote that threshold.

\section{A. Interfering transmitters}

In a chain network, while node 0 is receiving, any other simultaneously transmitting nodes must be some minimum distance away from node 0 to avoid interfering with node 0 's reception. Each potentially interfering transmitter must also be able to have its own transmissions successfully received. Since a chain network is symmetric and omnicentric, simultaneous transmitters are to be placed along the chain with equal transmitter separation (T-T) distance. Let $k$ denote that distance, in number of hops. With respect to node 0 receiving, it means that simultaneous transmitters will be at $k, 2 k \ldots n k \ldots$ and $-k,-2 k \ldots-n k \ldots$ Hence, the minimum $k$ indicates the best achievable spatial reuse.

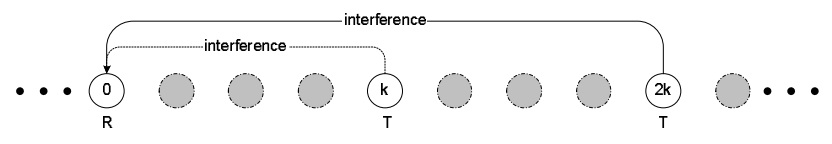

Figure 1. Interference accumulation on a chain

\section{B. Axial interference model}

In a chain network, the aggregate interference to a receiver comes from two symmetric groups, the right and the left, respectively. We define the accumulated interference in one direction as axial interference. Each axis includes an infinite number of interfering sources.

Recall the pathloss model in (1), let $D$ denote the inter-node distance. The axial interference $\left(P_{\text {axial }}\right)$ has a mean value given by:

$$
P_{\text {axial }}=A+10 \gamma \log _{10}(k \cdot D)+10 \log _{10}\left(\sum_{i=1}^{+\infty} i^{\gamma}\right)
$$

Note that the summation of the p-series, $\sum_{i=1}^{+\infty} i^{\gamma}$, is convergent only if $\gamma<-1$, which is typically true for any network scenario (refer to Table 1). Since no closed form for the $\mathrm{p}$ series summation is known for arbitrary $\gamma$, we derive an upper bound next.

Since function $i^{\prime}$ is monotonically decreasing with respect to $i$, so $\int_{n-1}^{n} i^{\gamma} d i>n^{\gamma} \cdot[(n+1)-n]$. Furthermore, adding the inequality from 1 to $\mathrm{n}$, we have $\int_{1}^{n} i^{\gamma} d i>\sum_{j=2}^{n} j^{\gamma}$. When $\gamma$ $<-1$, the LHS is in fact $\frac{(n+1)^{\gamma+1}-1}{\gamma+1}$. Letting $\mathrm{n} \rightarrow \infty$, we have an upper bound to the summation of the p-series,

$$
\sum_{i=1}^{+\infty} i^{\gamma}<\frac{\gamma}{\gamma+1}, \quad(\gamma<-1)
$$

Now the upper bound for the average axial interference is:

$$
P_{\text {axial }}<A+10 \gamma \log _{10}(k \cdot D)+10 \log _{10}\left(\frac{\gamma}{\gamma+1}\right)
$$

\section{Best achievable spatial reuse}

In a chain network, a node experiences interference from two axial groups of nodes. Therefore the total interference is twice the amount $(+3 \mathrm{db})$ of a single axial interference. Hence, the SIR (Signal-Interference Ratio, ignore noise for now) at a receiver is as follows,

$$
\begin{aligned}
& P_{\text {total }}=P_{\text {axial }}+3 d b \\
& S I R=P_{R x}(D)-P_{\text {total }}>-10 \gamma \log _{10}(k)-10 \log _{10}\left(\frac{\gamma}{\gamma+1}\right)-3 d b
\end{aligned}
$$

The above equation demonstrates three important properties of the SIR:

1. Varying the transmission power $\left(P_{0}\right.$, included in $A$ from (1) and (2)) will not change SIR. The aggregate interference scales the same as the received power of the intended signal with respect to transmission power.

2. Notice that $k$ is the separation distance between neighboring transmitters, in number of hops, hence the SIR depends on the relative T-T separation distance, not the absolute $\mathrm{T}-\mathrm{T}$ or $\mathrm{T}-\mathrm{R}$ separation distance.

3. SIR monotonically increases with respect to $k$.

Now let us factor in the ambient noise and consider SNIR. Since the ambient noise level remains unaffected by changing transmission power, and both signal and co-channel interference increase by the same factor, a higher transmission power actually increases SNIR. In other words, to reach the same SNIR threshold, a higher transmission power will allow the receiver to tolerate higher co-channel interference, i.e. a smaller $\mathrm{k}$. Hence, spatial reuse is improved.

In fact, in most dense narrowband wireless networks, ambient noise is negligible when comparing to co-channel interference. Hence in the rest of analysis, we use SIR to approximate SNIR.

Notice that runtime behavior of wireless communication reflects a stochastic process. The receiving strength characterized by the pathloss model in (1) is expressed as a random variable, as is the runtime SIR. Nonetheless, since the success probability of a communication strongly depends on the mean value of SIR, this paper will emphasize the condition which leads to average SIR being greater than the threshold $S_{0}$. Since the lower bound given in (5) is for the 
mean value of SIR, the following is the sufficient condition for successful communication:

$$
S_{0} \leq-10 \gamma \log _{10}(k)-10 \log _{10}\left(\frac{\gamma}{\gamma+1}\right)-3 d b
$$

which leads to the following lower bound for $k$,

$$
k \geq\left[2 \cdot\left(\frac{\gamma}{\gamma+1}\right) \cdot 10 S_{0} / 10\right]^{-1 / \gamma}
$$

Essentially, (7) indicates the minimum $k$ value which reflects the best achievable spatial reuse in a chain network.

\section{Regular 2-D Networks}

Consider a regular 2-D network of uniform density with an infinite number of nodes; $k$ denotes the separation distance between neighboring simultaneous transmitters measured in number of hops. For a given receiver at the center, at most 6 simultaneous transmitters can exist on the $k$-hop radius circle as shown in Figure 2(a); the locations of these transmitters form the vertices of a hexagon whose edge is of length $k$ hops. Extending this analysis, simultaneous transmitters will form an infinite hexagon tessellation overlaying the 2-D network as shown in Figure 2(b). All the transmitters reside at the vertices where the edge of each hexagon is $k$ hops long for a reference receiver located at the center.

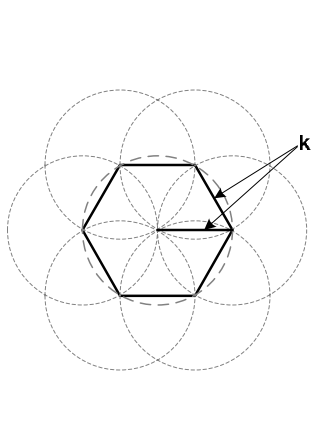

(a)

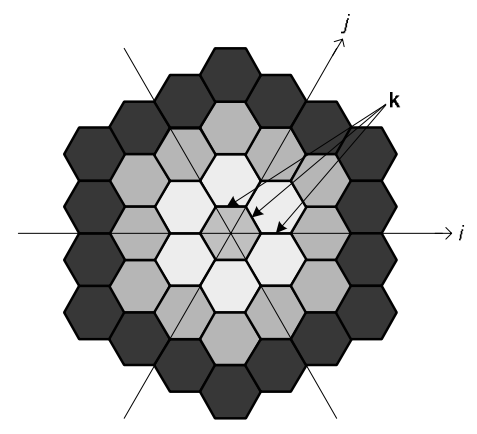

(b)

Figure 2. Transmitter layout in 2-D network

Given this regular 2-D network topology, the accumulated interference at a receiver is

$$
\begin{aligned}
& P_{\text {total }}=7.8 d b+A+10 \gamma \log _{10}(k \cdot D) \\
& +10 \log _{10}\left[\sum_{i=0}^{+\infty} \sum_{j=1}^{+\infty}\left(i^{2}+j^{2}+i j\right)^{\gamma / 2}\right]
\end{aligned}
$$

Using the same upper bound for p-series as for the 1-D case, the aggregate interference in a 2-D symmetric network is upper-bounded by

$$
P_{\text {total }}<7.8 d b+A+10 \gamma \log _{10}(k \cdot D)+10 \log _{10}\left(\frac{\gamma+1}{\gamma+2}\right)
$$

Hence, the SIR has a lower bound of

$$
S I R>-10 \gamma \log _{10} k-10 \log _{10}\left(\frac{\gamma+1}{\gamma+2}\right)-7.8 d b
$$

Again, the SIR has the same three properties as in a 1-D chain network. In noise limited scenarios, a higher transmission power will improve SNIR and hence improve spatial reuse. When interference dominates, the minimum $k$ value for a 2-D symmetric network is

$$
k \geq\left[6 \cdot\left(\frac{\gamma+1}{\gamma+2}\right) \cdot 10^{S_{0} / 10}\right]^{-1 / \gamma}
$$

Results for various network topologies and propagation environments have shown the dependence of spatial reuse on these key factors.

\section{Quantitative Results}

\section{A. Spatial reuse}

In our analysis, spatial reuse is characterized by the separation distance between simultaneous transmitters, i.e. the value of $k$, in number of hops. We have identified that spatial reuse is determined by the receiver SIR requirements, the propagation path loss and the network topology.

Figure 3 plots the spatial reuse (as given by equation 7 ) in a chain network with respect to pathloss exponent for three SIR threshold: $8 \mathrm{~dB}, 13 \mathrm{~dB}$ and $18 \mathrm{~dB}$, respectively. In a typical $802.11 \mathrm{~b}$ (2.4GHz ISM band) network, $18 \mathrm{db}$ is considered good channel quality which can sustain $11 \mathrm{Mbps}$ data rate. For $\gamma=-2$, representing a typical free space environment, the T-T separation distance will be around 13.

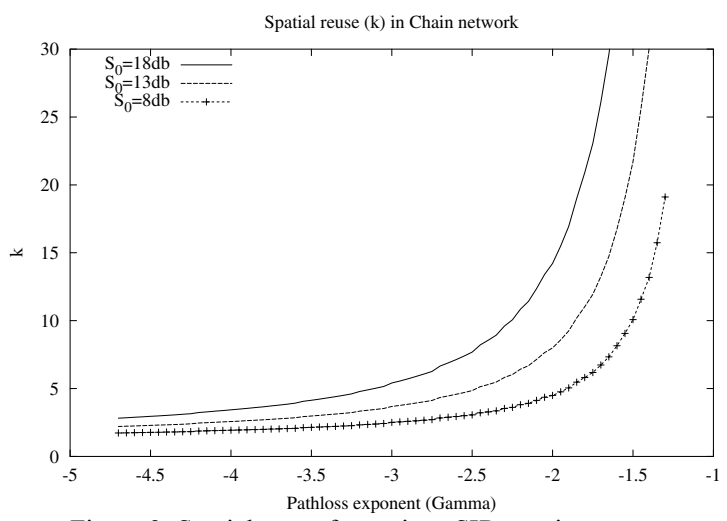

Figure 3. Spatial reuse for various SIR requirements

Likewise, Figure 4 plots the spatial reuse for 2-D symmetric network. For comparison, the result for a chain network at $8 \mathrm{db}$ is also included in this figure. 
As demonstrated previously, calculation of the spatial reuse factor $\mathrm{k}$ centers around interference estimation which is based on using the upper bound for p-series summation in (3). To evaluate the accuracy of the approximation, in Figure 5 the spatial reuse value resulting from use of our $\mathrm{p}$ series upper bound is compared against the baseline value. The latter is an accurate "brute-force" numerical computation of the p-series until the relative error is less than 1e-7. As shown, the lower bound adopted for the summation of the p-series matches well against the baseline over the range of interest.

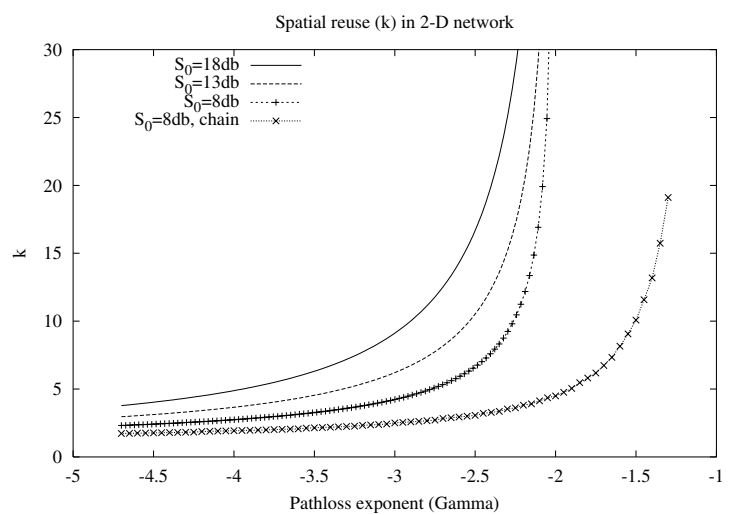

Figure 4. Spatial reuse in 2-D symmetric network

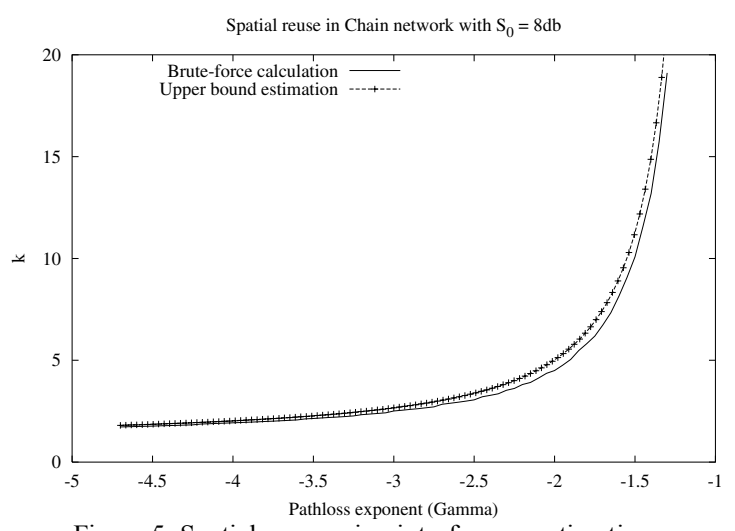

Figure 5. Spatial reuse using interference estimation

\section{B. Comparison with cellular analysis}

In cellular networks, the co-channel spatial reuse factor is characterized by $R$, the co-channel reuse ratio [21]. Using the notation in this paper, the relationship between $R$ and the SIR threshold at the receiving node is as follows,

$$
R=\left[6 \cdot\left(10^{S_{0} / 10}\right)\right]^{-1 / \gamma}
$$

Essentially, the interference model in a cellular network assumes that interference sources are limited to the base stations of the 6 nearest co-channel cells and ignores contributions from more distant base stations. So the model uses only the six first tier terms for the summation of the entire $\mathrm{p}$ - series in equation (3). Figure 6 compares the accuracy of the two models. The baseline interference is calculated using brute-force iterative computation on equation (8), and our interference estimation is from (9). As the figure shows, both results are within $5 \%$ of the real interference value for outdoor environment, where the typical pathloss exponent is less than -4 . However, when the pathloss exponent becomes larger than -4 , the accuracy of the cellular approximation starts to suffer. In [21], the author cited several measurements of pathloss exponent for various indoor environments such as retail stores, factory plants, office buildings, etc., where the pathloss exponents were found to range from -1.8 to -3.3 . As demonstrated in Figure 6, while the cellular result can be more than $25 \%$ off the reference value, our approximation maintains 5\% accuracy for these indoor environments.

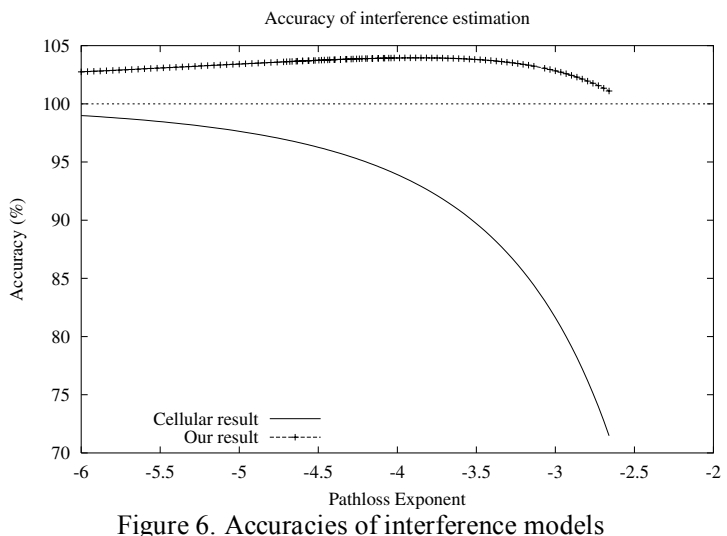

VI. Related Work

Cellular networks with planned frequency reuse partition the coverage area into cells based on hexagon tessellation [18][21]. Each cell is served by a base station and a subset of all available radio channels are allocated either statically to the base stations, or dynamically "traded" amongst base stations. A user device is instructed by the base station for the appropriate channel to communicate [12].

In contrast, the lack of engineered infrastructure and the random topology of ad-hoc networks lead to new implications for spatial reuse and its consequent impact on capacity of wireless ad-hoc networks. In [2], the performance of various routing algorithms for wireless ad-hoc networks were compared in a simulation environment built around ns2 based on a static range model [7]. In [16], the authors examined the throughput capacities of $802.11 \mathrm{~b}$ wireless adhoc networks [11], through theoretical studies and ns-2 simulations. They found that the 802.11 MAC protocol yields throughput that is markedly less than is possible with an optimal schedule, either because it failed to detect an idle link immediately, or it generates too much local collision. This conforms to our position that cross-layer interaction among the network stack may significantly affect the overall 
network capacity. In [9], the authors investigated how node mobility affects the capacity of the network, and they found out that allowing random node movement in the network can actually increase the overall capacity of the network, if arbitrary delay is acceptable. In [10], the authors took an information theory approach to study two types of wireless ad-hoc network environments: arbitrary network and random network. Network transport capacity was measured by the number of bits transmitted for unit distance in unit time and it was shown that (a) the capacity of an arbitrary network is of order $\Theta(\sqrt{n})$ where $\mathrm{n}$ is the node density whereas (b) the random network has a capacity of $\Theta(\sqrt{n / \log (n)})$.

\section{Conclusion}

The total transport capacity of a wireless ad-hoc network is proportional to the number of simultaneous communications that can be accommodated in the network, which ultimately is determined by co-channel spatial reuse. In this paper, we investigated the spatial reuse in dense wireless 1-D chain network topologies and 2-D symmetric network topologies. We used a link layer derived interference model for estimating the worst-case interference at the physical layer to derive the minimal reuse distance. The lower bounds of transmitter separation distances demonstrate that spatial reuse is affected by the type of the radio, the channel path loss exponent and the network topology and underscores the limitations of the static range model used in a large majority of work in this area.

Furthermore, increasing transmission power on homogeneous nodes improves spatial reuse only in ambient noise limited scenario. When the reception is dominated by cochannel interference, varying transmission power has minimal impact on spatial reuse. Nonetheless, by reducing the inter-node distance, more non-interfering communications can be accommodated in the same area. Propagation environments with higher path loss exponents allow better spatial reuse.

\section{References}

[1] Berkeley Wireless Research Center, DARPA Pico Radio Project Proposal, October 1999, http://bwrc.eecs.berkeley.edu.

[2] Josh Broch, David A. Maltz, David B. Johnson, Yih-Chun Hu and Jorjeta Jetcheva. A Performance Comparison of Multi-Hop Wireless Ad-Hoc Network Routing Protocols. Proceedings ACM/IEEE MobiCom'98, Dallas 1998.

[3] David Cheung and Cliff Prettie. A Path Loss Comparison Between the $5 \mathrm{GHz}$ UNII Band (802.11a) and the 2.4GHz ISM Band (802.11b). Technical report, Intel Labs, January 2002.

[4] I. Chlamtac and S. Kutten. A spatial reuse TDMA/FDMA for mobile multi-hop radio networks. Proceedings of IEEE INFOCOM 1985.

[5] Deborah Estrin, Ramesh Govindan, John Heidemann and Satish Kumar. Next century challenges: scalable coordination in sensor networks. ACM MobiCom 1999.
[6] Shlomi Dolev, Evangelos Kranakis, Danny Krizanc and David Peleg. Bubbles: adaptive routing scheme for high-speed dynamic networks. SIAM Journal on Computing, 1999.

[7] Kevin Fall and Kannan Varadhan. ns Notes and Documentation. Technical report, UC Berkeley, LBL, USC/ISI, and Xerox PARC, November 1997.

[8] Jimmi Gronkvist. Assignment Methods for Spatial Reuse TDMA. IEEE MobiHOC 2000.

[9] Matthias Grossglauser and David Tse. Mobility Increases the Capacity of Ad-hoc Wireless Networks. Proceedings IEEE Infocom, April 2000.

[10] Piyush Gupta and P.R. Kumar. The Capacity of Wireless Networks. IEEE Transactions on Information Theory, 46(2):388-404, March 2000.

[11] IEEE Computer Society LAN MAN Standard Committee. Wireless LAN Medium Access Control (MAC) and Physical Layer (PHY) Specifications. IEEE Std. 802/11-1997. New York, 1997.

[12] I. Katzela and M. Naghshineh. Channel Assignment Schemes for Cellular Mobile Telecommunication Systems: A Comprehensive Survey. IEEE Personal Communications, June 1996.

[13] Lakshman Krishnamurthy, Steven Conner, Mark Yarvis, Jasmeet Chhabra, Carl Ellison, Chuck Brabenac and Ernest Tsui. Meeting the Demands of the Digital Home with High-Speed Multi-Hop Wireless Networks. Intel Technology Journal, Vol.6, Issue 4, 2002.

[14] W.C.Y. Lee. Elements of Cellular Mobile Radio systems. IEEE Trans. on Vehicular Tech., Vol. VT-35, No.2, May 1986.

[15] W.C.Y. Lee. Key Elements in Designing a CDMA System. IEEE Trans. Vehicular Tech. Vol.44, No.3, June 1994.

[16] Jinyang Li, Charles Blake, Douglas S.J. De Couto, Hu Imm Lee and Robert Morris. Capacity of Ad Hoc Wireless Networks. Proceedings of ACM SIGMOBILE, July 2001. Rome, Italy.

[17] R. Nelson and L. Kleinrock. Spatial-TDMA: A collision-free multihop channel access protocol. IEEE Trans. Communication, vol.33, no.9, September 1985

[18] J. Oeting. Cellular Mobile Radio - An Emerging Technology. IEEE Comm. Magazine, pp.10-15, November 1983.

[19] G.J. Pottie and W.J. Kaiser. Wireless Integrated Networked Sensors. Communications of the ACM, 43(5):51-58, May 2000.

[20] Theodore S. Rappaport and Laurence Milstein. Effects of Radio Propagation Path Loss on DS-CDMA Cellular Frequency Reuse Efficiency for the Reverse Channel. IEEE Trans. Vehicular Tech., Vol.41, No.3, August 1992.

[21] Theodore S. Rappaport. Wireless Communications, Principles and Practices, $2^{\text {nd }}$ Ed. Prentice Hall, 2002. ISBN 0-13-042232-0.

[22] Theodoros Salonidis, Pravin Bhagwat, Leandros Tassiulas and Richard LaMaire. Distributed topology construction of Bluetooth personal area networks. IEEE INFOCOM 2001.

[23] Timothy J. Shepard. A Channel Access Scheme for Large Dense Packet Radio Networks. Proceedings ACM SIGCOMM, pages 219-230, August 1996.

[24] Mark Yarvis, Steven Conner, Lakshman Krishnamurthy, Jasmeet Chhabra, Brent Elliot, and Alan Mainwaring. Real-World Experiences with an Interactive Ad Hoc Sensor Network. Proceedings of the International Workshop on Ad hoc Networking (IWAHN 2002), Vancouver, British Columbia, Canada, August 2002. 